\title{
A retrospective analysis of the microbiology of diabetic foot infections at a Scottish tertiary hospital
}

\author{
Katherine E. Macdonald ', Crispin Y. Jordan², Emma Crichton ${ }^{3}$, Judith E. Barnes ${ }^{3}$, Gillian E. Harkin³,
} Lesley M. L. Hall ${ }^{3}$ and Joshua D. Jones ${ }^{1,4^{*}}$ (i)

\begin{abstract}
Background: This study represents the first Scottish retrospective analysis of the microbiology of diabetic foot infections (DFIs). The aims were to compare the microbiological profile of DFls treated at a Scottish tertiary hospital to that in the literature, gather data regarding antimicrobial resistance and investigate potential trends between the microbiological results and nature or site of the clinical sample taken and age or gender of the patients.

Methods: A retrospective analysis of wound microbiology results was performed, data were obtained from one multidisciplinary outpatient foot clinic during the 12 months of the year 2017. Seventy-three patients and 200 microbiological investigations were included. In cases of soft tissue infection, the deepest part of a cleansed and debrided wound was sampled. In cases of osteomyelitis a bone biopsy was obtained. Factors influencing the pattern of microbial growth or prevalence of Staphylococcus aureus were investigated.

Results: Of the 200 microbiological investigations, 62\% were culture positive, of which $37.9 \%$ were polymicrobial and $62.1 \%$ monomicrobial. Among the monomicrobial results $(n=77)$, most were Gram positive isolates (96.1\%) and the most frequently isolated bacteria was S. aureus (84.4\%). No methicillinresistant $S$. aureus was reported. The prevalence of $S$. aureus in DFIs was associated with increasing age $(p=0.021)$, but no evidence of association with gender, anatomical sample site or sample material was found.

Conclusion: The microbiological profile of DFIs in Scotland resembles that reported elsewhere in the UK. In this context, Gram positive organisms, primarily S. aureus, are most frequently isolated from DFls. The S. aureus isolates identified were largely susceptible to antibiotic therapy. An association between increasing patient age and the prevalence of $\mathrm{S}$. aureus in DFls was observed.
\end{abstract}

Keywords: Diabetic foot infection, Microbiology, Retrospective, Staphylococcus aureus

\footnotetext{
* Correspondence: josh.jones@ed.ac.uk

${ }^{1}$ Edinburgh Medical School: Biomedical Sciences, Infection Medicine,

University of Edinburgh, Chancellor's Building, 49 Little France Crescent, Edinburgh EH16 4SB, UK

${ }^{4}$ ZJU-UoE Institute, Zhejiang University School of Medicine, International

Campus, Zhejiang University, 718 East Haizhou Road, Haining, Zhejiang

314400, People's Republic of China

Full list of author information is available at the end of the article
}

(c) The Author(s). 2020 Open Access This article is licensed under a Creative Commons Attribution 4.0 International License, which permits use, sharing, adaptation, distribution and reproduction in any medium or format, as long as you give appropriate credit to the original author(s) and the source, provide a link to the Creative Commons licence, and indicate if changes were made. The images or other third party material in this article are included in the article's Creative Commons licence, unless indicated otherwise in a credit line to the material. If material is not included in the article's Creative Commons licence and your intended use is not permitted by statutory regulation or exceeds the permitted use, you will need to obtain permission directly from the copyright holder. To view a copy of this licence, visit http://creativecommons.org/licenses/by/4.0/. The Creative Commons Public Domain Dedication waiver (http://creativecommons.org/publicdomain/zero/1.0/) applies to the data made available in this article, unless otherwise stated in a credit line to the data. 


\section{Background}

Diabetes mellitus, herein diabetes, is the most common metabolic disease in the world. In 2014 there were an estimated 422 million adults living with diabetes, and the global prevalence continues to increase, with a projected 592 million people living with the condition by 2035 [1, $2]$. Around one third of patients with diabetes will develop a diabetic foot ulcer (DFU) and approximately half of these will become infected [3]. DFUs develop secondary to a variety of factors, including peripheral neuropathy and peripheral arterial disease [4]. Diabetes in Scotland represents a significant burden, with nearly 300,000 people registered as having the condition in 2017 [5]. Many of these individuals (44.2\%) have had diabetes for more than 20 years [5], a significant risk factor for the development of a DFU [6].

Infected DFUs are known as diabetic foot infections (DFIs) and carry a high burden morbidity and increased risk of mortality [3]. The microorganisms most commonly isolated from DFIs are aerobic Gram positive bacteria, predominantly Staphylococci $[7,8]$. The prevalence of methicillin resistant Staphylococcus aureus (MRSA) in DFIs is considered to be $15-30 \%$ [8, 9]. Other bacterial genera commonly found in DFIs include Streptococci, Enterococci, Enterobacteriaceae, and Pseudomonas [3]. These infections are often polymicrobial and understanding the melange of species present can help inform clinical practice. Treatment for DFIs consists of wound care and antibiotics. The chronic and recalcitrant nature of these infections typically requires the administration of long courses of antibiotics, which may contribute to antimicrobial resistance. The efficacy of haematogenously distributed antibiotics may be further hampered by peripheral arterial disease [10].

An understanding of the microbiology of DFIs is important to inform clinical practice, offer insight into local microbiological trends and direct novel therapeutic strategies. To date, there has been just one small prospective study of the microbiology of DFIs in Scotland [11]. To supplement this, we therefore undertook the first Scottish retrospective analysis of the microbiology of DFIs. All DFI patients treated at an outpatient clinic at the Queen Elizabeth University Hospital (QEUH) in Glasgow during the 12 months of 2017 were included. The objectives were to compare the local microbiological profile to that in the literature, gather data regarding antimicrobial resistance and investigate potential relationships between the microbiological results and nature or site of the clinical sample taken and age or gender of the patients.

\section{Methods}

This retrospective cohort study was undertaken in the multidisciplinary diabetic foot service at the tertiary Queen Elizabeth University Hospital in Glasgow, Scotland. The results of all microbiological investigations ordered by one multidisciplinary outpatient foot clinic during the 12 months of the year 2017 were retrieved by members of the direct care team. The age (years) and gender of each patient were recorded. Further information about the diabetes type of these patients could not readily be obtained due to the division of patient data between discrete electronic platforms. Microbiological investigations were undertaken, prior to the administration of antibiotics. Wound sampling was performed according to the National Institute for Health and Clinical Excellence guidelines for diabetic foot problems 1.6.1 [12]. All samples were taken from the base of a cleansed and debrided wound. In cases of soft tissue infection, a deep wound swab was taken from the debrided ulcer base. Bone biopsies were obtained in cases of suspected osteomyelitis. For all investigations, care was taken to prevent contamination with skin flora and provide clinically relevant results to inform patient care. All microbiological investigations were performed according to the UK Standards for Microbiology Investigations and local microbiological guidelines. Deep tissue swabs were placed on Amies transport medium with charcoal and sent to the Queen Elizabeth University Hospital Microbiology Department. Bone biopsies were placed in a sterile universal container. All samples were cultured for aerobic and anaerobic microorganisms, pure cultures were obtained and subjected to antibiotic sensitivity testing. The presence of coagulase negative Staphylococci in deep tissue swabs is routinely presumed to indocate skin flora and is not reported as significant growth. The following data were recorded for each result: investigation site (broadly divided into foot, toe or ankle), material sampled (bone biopsy or deep tissue swab) and general bacterial growth (no significant growth, monomicrobial or polymicrobial). For monomicrobial plates, the species identified were recorded, alongside any available antimicrobial sensitivity data. All data were anonymised before secure transmission from NHS Greater Glasgow \& Clyde to the University of Edinburgh. This study was performed as a quality improvement study and Caldicott Guardian approved by NHS Greater Glasgow and Clyde.

Statistical analyses were performed using RStudio (version 1.1456) and Microsoft Excel. In R, the "lme4" and "lmerTest" packages were utilised $[13,14]$. Four generalised linear mixed-effects model were run, taking into account the random effects of patients, to analyse the statistical significance of each parameter recorded in turn (the four independent variables: age, gender, investigation site and material sampled) in influencing the prevalence of $S$. aureus. The dependent variable in our models was the binomial response of whether an investigation signalled a $S$. aureus infection ('yes' vs. 'no'); therefore, our models assumed a binomial distribution. We report our model results in terms of the 'log odds' that a given clinical sample is infected, $\log (p /(1-p))$, 
where $p$ equals the probability that a sample is infected. Therefore, $p /(1-p)$ represents the odds of infection. For example, if the probability of being infected is 0.8 , then the odds of being infected equals four (0.8/0.2). The 'Diagnostics for HierArchical Regression Models' (DHARMa) package was used to check the residuals for the mixed effects model [15]. Microsoft Excel was used to perform descriptive statistics on age, clinical microbiology and antimicrobial resistance.

\section{Results}

\section{Study population}

Seventy-three patients from one multi-disciplinary diabetic foot outpatient clinic had one or more microbiological investigations during 2017 and were therefore included in this retrospective study. In cases of soft tissue infection, deep tissue swabs were taken from the cleansed and debrided ulcer base. Bone biopsies were obtained in suspected cases of osteomyelitis. A total of 201 microbiological investigations were performed, however microbiological data for one monomicrobial deep tissue swab result were missing and this result was excluded; therefore, 200 microbiolocial results were included in this analysis. The study population comprised 56 males and 17 females. The age of the patients ranged from 28 to 94 , with an average age ( \pm standard deviation) of $63.8 \pm 12.8$ years (Fig. 1).

The effect of gender, swab site and sample material on the nature of the swab result

Of the 200 microbiological investigations, $62 \%$ were culture positive, $37.9 \%$ of which were polymicrobial and
$62.1 \%$ were monomicrobial. The distribution of the 200 results by gender, anatomical sample site and material sampled is shown in Table 1. About a third (38.1\% male; $37.5 \%$ female) of investigations did not yield significant growth (i.e. these samples were sterile or represented presumed skin flora and were not reported by the Microbiology Department as significant). Among those infected, the majority had monomicrobial infections. A high proportion of monomicrobial results was also observed among clinical samples taken from foot $(42.4 \%)$ or toe $(36.2 \%)$ infections and from deep tissue (39.3\%) swabs. Few clinical samples were obtained from the ankle $(n=3)$ and there were few bone biopsy $(n=9)$ results available. A Chi-Square test could not be performed with these data because of violation of the assumption of independence as some patients provided multiple sample results.

\section{The microbiological profile of diabetic foot infection}

The mean number of microbiological results per patient was 2.74 with a range of one to nine results and a mode of one. In some cases, the results of patients who had had multiple investigations were identical. However, clinical staff at QEUH advised that microbiological investigations are only routinely taken at the start of an infectious episode, with patients then being treated until clinical resolution of infection. Additional investigations in the same patient therefore likely reflect a new episode of infection. Therefore, all results were included in the analyses.

Among the results, 124 were positive for microbial growth, representing 1.70 positive cultures per patient.

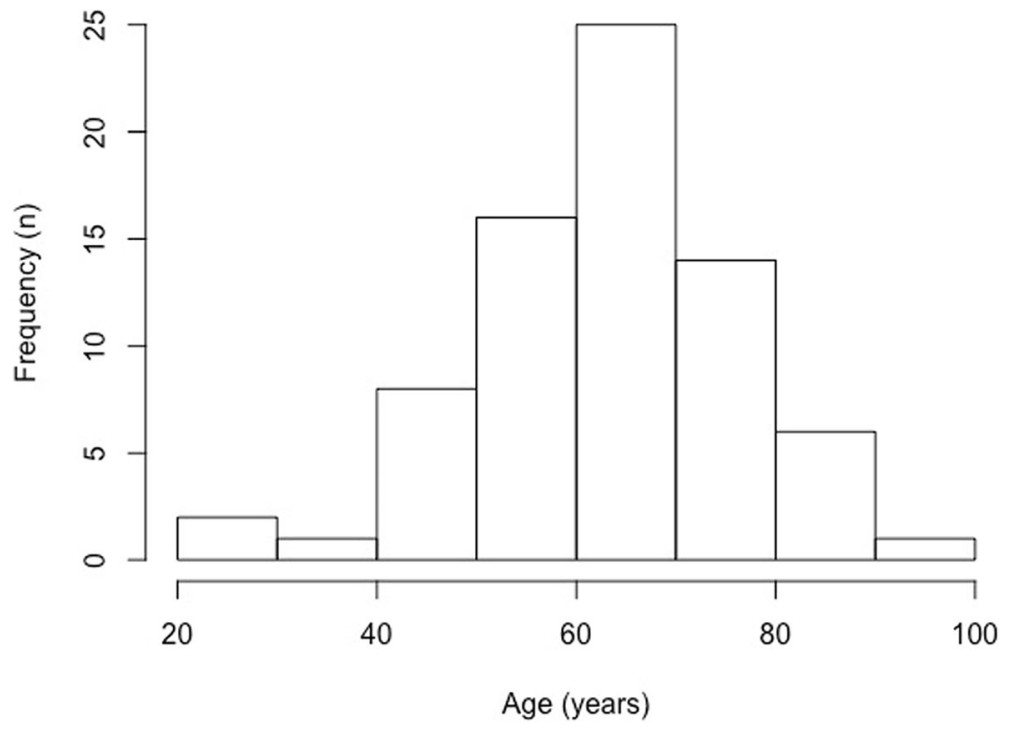

Fig. 1 The age distribution of the diabetic foot patients included in this study $(n=73)$ 
Table 1 The relationship between gender, sample site and sample type and the pattern of microbial growth observed

\begin{tabular}{|c|c|c|c|c|}
\hline & \multicolumn{3}{|c|}{ Pattern of microbial growth } & \multirow{2}{*}{$\begin{array}{l}\text { Total } \\
n \\
(100 \%)\end{array}$} \\
\hline & $\begin{array}{l}\text { Monomicrobial } \\
\mathrm{n}(\%)\end{array}$ & $\begin{array}{l}\text { Polymicrobial } \\
\text { n (\%) }\end{array}$ & $\begin{array}{l}\text { No significant growth } \\
\mathrm{n}(\%)\end{array}$ & \\
\hline All clinical samples & $77(38.5 \%)$ & $47(23.5 \%)$ & $76(38.0 \%)$ & 200 \\
\hline Male & $62(36.9 \%)$ & $42(25.0 \%)$ & $64(38.1 \%)$ & 168 \\
\hline Female & 15 (46.9\%) & $5(15.6 \%)$ & $12(37.5 \%)$ & 32 \\
\hline Ankle & $0(0.0 \%)$ & $0(0.0 \%)$ & $3(100 \%)$ & 3 \\
\hline Foot & 39 (42.4\%) & $22(23.9 \%)$ & $31(33.7 \%)$ & 92 \\
\hline Toe & $38(36.2 \%)$ & $25(23.8 \%)$ & $42(40.0 \%)$ & 105 \\
\hline Bone biopsy & $2(22.2 \%)$ & $6(66.7 \%)$ & $1(11.1 \%)$ & 9 \\
\hline Deep tissue swab & 75 (39.3\%) & $41(21.5 \%)$ & 75 (39.3\%) & 191 \\
\hline
\end{tabular}

An exact mean number of isolates per patients could not be calculated given the polymicrobial nature of some results. The microorganisms isolated are detailed in Table 2.

Excluding fungal, mixed and samples without significant growth, there were 77 monomicrobial results, of which $96.1 \%$ were Gram positive bacterial

Table 2 The microorganisms reported from diabetic foot wound cultures $(n=200)$

\begin{tabular}{lll}
\hline & $\begin{array}{l}\text { Number of microbiological } \\
\text { results }\end{array}$ \\
\hline Gram positive & Staphylococcus aureus & 65 \\
Staphylococcus epidermidis & 1 \\
Staphylococcus & 2 \\
lugdenensis & \\
Group B Streptococcus & 2 \\
Group C Streptococcus & 1 \\
Group F Streptococcus & 1 \\
Group G Streptococcus & 1 \\
Streptococcus constellatus & 1 \\
Total Gram positive & $\mathbf{7 4}$ \\
Enterobacter hormaechei & 1 \\
Escherichia coli & 1 \\
Pseudomonas aeruginosa & 1 \\
Total Gram negative & $\mathbf{3}$ \\
Total monomicrobial & $\mathbf{7 7}$ \\
swabs & \\
Yeasts & 1 \\
Mixed aerobic & 40 \\
Mixed anaerobic & 6 \\
Total positive swabs & 124 \\
No significant growth & 76 \\
Total & 200 \\
\hline
\end{tabular}

species and $3.9 \%$ were Gram negative. On a per patient basis $(n=73)$, only $4.1 \%$ of individuals ever grew a Gram negative species, and 43 patients tested positive on at least one occasion for a Gram positive bacterium.

The prevalence of $S$. aureus in diabetic foot infections was influenced by age but not gender, anatomical sample site or sample material

Staphylococcus aureus was found in $32.5 \%$ of all results $(n=200)$ and accounted for $84.4 \%$ of the 77 monomicrobial results. We investigated whether the prevalence of $S$. aureus was related to gender, anatomical sample site or sample material using separate generalised linear models with mixed effects for each variable (Table 3). We found that the prevalence of $S$. aureus was not influenced by gender $(p=0.249)$, anatomical site $(p=0.202)$ or sample material $(p=0.556)$. A fourth generalised linear mixed-effects model indicated that the prevalence of $S$. aureus increased with age $(p=0.0211)$. The significance of this effect persisted following exclusion of the three youngest patients (potential outliers; aged 28, 29 and 36; $p=$ 0.0451). We found that the mean change in log odds of being infected with $S$. aureus increases by $0.0372 \pm$ 0.0161 annually (equivalently, each year, the odds of infection increase multiplicatively by exp.(0.0372)), with an intercept of -3.24 . For example, at age 40 the predicted probability (i.e., out of 1 ) of having a $S$. aureus positive DFI is 0.15 . Once a patient reaches age 60 , this increases to 0.27 . Finally, by age 80 the predicted probability reaches 0.43 . Thus, with increasing age the probability of having a $S$. aureus positive diabetic foot infection becomes more likely. Analyses with DHARMa indicated that our data met the assumptions of our model. To visualise this effect, we calculated the proportion of $S$. aureus positive results per patient, which ranged from 0 (if all swabs were 
Table 3 The effect of gender, anatomical swab site and sample material on the prevalence of S. aureus among DFI patients

\begin{tabular}{|c|c|c|c|}
\hline \multicolumn{4}{|c|}{ The prevalence of S. aureus in: } \\
\hline \multicolumn{4}{|c|}{$\begin{array}{ll}\text { All results } \\
(n=200)\end{array}$} \\
\hline \multicolumn{4}{|c|}{$\begin{array}{l}32.5 \% \\
(n=200)\end{array}$} \\
\hline Male & $\begin{array}{l}30.3 \% \\
(n=168)\end{array}$ & $\begin{array}{l}82.3 \% \\
(n=62)\end{array}$ & - \\
\hline Female & $\begin{array}{l}43.8 \% \\
(n=32)\end{array}$ & $\begin{array}{l}93.33 \% \\
(n=15)\end{array}$ & $(p=0.249)$ \\
\hline Ankle & $\begin{array}{l}0 \% \\
(n=3)\end{array}$ & $\begin{array}{l}0 \% \\
(n=3)\end{array}$ & - \\
\hline Foot & $\begin{array}{l}38 \% \\
(n=92)\end{array}$ & $\begin{array}{l}78.9 \% \\
(n=38)\end{array}$ & - \\
\hline Toe & $\begin{array}{l}28.5 \% \\
(n=105)\end{array}$ & $\begin{array}{l}89.7 \% \\
(n=39)\end{array}$ & $(p=0.202)$ \\
\hline Bone biospy & $\begin{array}{l}22.2 \% \\
(n=9)\end{array}$ & $\begin{array}{l}100 \% \\
(n=2)\end{array}$ & - \\
\hline Deep tissue swab & $\begin{array}{l}33.3 \% \\
(n=191)\end{array}$ & $\begin{array}{l}84 \% \\
(n=75)\end{array}$ & $(p=0.556)$ \\
\hline
\end{tabular}

negative) to 1 (if all results were positive), and plotted these data against the age for each individual (Fig. 2).

\section{Antimicrobial resistance of $S$. aureus}

Finally, we examined the available antimicrobial sensitivity data. For some patients, antimicrobial resistance was investigated at multiple clinic visits. A total of 62 microbiological results from $38 \mathrm{~S}$. aureus positive patients were tested for flucloxacillin resistance, with $95.2 \%$ of $S$. aureus isolates found to be sensitive. Clarithromycin sensitivity was investigated for $62 S$. aureus isolates obtained from 38 patients, two of which were not tested for flucloxacillin resistance; of these $72.6 \%$ of $S$. aureus isolates were found to be sensitive. A small number of other S. aureus antibiotic sensitivity investigations were requested, with 95\% sensitivity recorded against doxycycline $(n=20)$ and $100 \%$ sensitivity to penicillin $(n=8)$, clindamycin $(n=4)$ and vancomycin $(n=1)$. Notably, no MRSA was detected in this study.

\section{Discussion}

This study represents the first retrospective analysis of the microbiology of DFIs undertaken in Scotland. The aim was to investigate whether the microbiological profile of diabetic foot infections at a Scottish hospital was comparable to previously published reports. The literature suggests that in the UK Staphylococci, particularly S. aureus, are most commonly isolated from DFIs [1619], and the findings of this study support this. A previous prospective culture-based microbiological analysis of 20 Scottish DFI samples also identified $S$. aureus as the most common microorganism, present in $40 \%$ of samples [11]. Although widely associated with DFIs, Staphylococci often exist as commensal organisms, particularly in the nose and throat, and the prevalence of $S$. aureus carriage is thought to be around 30\% [20]. This is supported by UK data, including a community-based study which showed a $S$. aureus carriage rate of 28\% [21] and a prevalence of $41 \%$ among Royal Marine recruits [22]. Staphylococci often colonise the skin [23, 24], and, despite following guidelines for the investigation of wound microbiology, we therefore cannot exclude the possibility that some Staphylococcal cultures represent skin commensals. For this reason coagulase-negative Staphylococci are locally not routinely reported for deep tissue swabs. Other major pathogens associated with DFIs include Enterococci, Streptococci, Escherichia coli and Pseudomonas aeruginosa [25]. However, these pathogens either occurred at a relatively low frequency or were absent from our data. It has been reported that Gram positive organisms are often more frequently isolated from DFIs in Western nations, with Gram

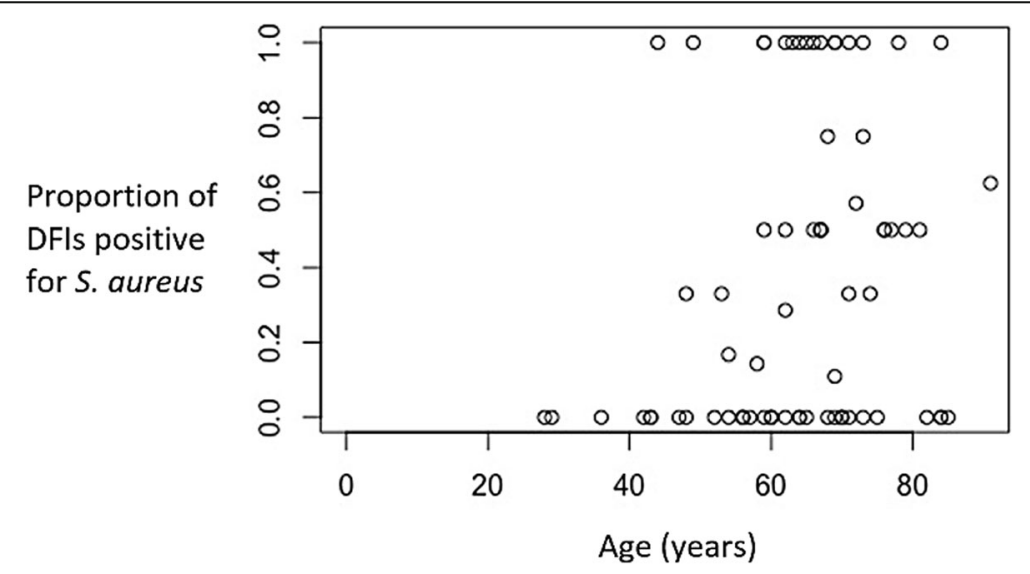

Fig. 2 The effect of age on the prevalence of S. aureus among diabetic foot infections $(n=73)$ 
negatives more frequently isolated from warmer climates, including Asia and Africa. The reasons for this remain unclear but could reflect a variety of environmental, clinical or personal factors [26]. The frequency of samples with no significant growth (38\%) is broadly comparable with that found in similar studies in the UK $[16,19]$, and similar to that reported for the only other Scottish analysis of the microbiology of DFIs (45\%) performed at the same hospital [11].

Given the potential breadth of associated factors and limited sample size, it was not surprising that our descriptive analysis of gender and anatomical sample site did not reveal any clear association with the broad pattern of microbiological growth observed. Although analysis of these data was limited by violation of an assumption of the Chi-Square test, the difference between the microbiological patterns reported from bone biopsies and deep tissue swabs was intriguing, but limited by the sample size. A higher frequency of polymicrobial results from bone samples could suggest that these patients had deeper and potentially more complex and chronic infections. More broadly, there are conflicting reports of the concordance of bone and tissue samples $[27,28]$.

Young age and male gender are among a range of factors sometimes considered to increase the carriage of $S$. aureus carriage in the community, although mixed results have been observed [20, 29]. While these factors may affect carriage rates, this study found no evidence that gender is a factor that contributes to the chance of having a $S$. aureus positive DFI. There was also no evidence to suggest that sample site or material were associated with a higher rate of $S$. aureus infection. This study therefore suggests that the rate of $S$. aureus positive DFIs may be independent of these factors, however these findings are somewhat limited by the statistical power. In general, the prevalence of commensal $S$. aureus colonisation is considered to decline with increasing age, including among hospital patients [20, 29]. However, this study found that the prevalence of $S$. aureus in DFIs was in fact associated with increasing patient age. The reasons for this are unclear, but it may reflect sample size or underlying temporal trends in the clinical state of these patients.

Antimicrobial resistance is a common finding in DFIs, with meta-analyses estimating the global prevalence of methicillin-resistant $S$. aureus in DFIs to be $15-30 \%$ [8, 9]. Previous studies in the UK have reported higher rates, ranging from 19.9 to $57.9 \%$ [16-19]. Reassuringly this analysis did not report the isolation of any MRSA and, where investigated, a high degree of sensitivity to flucloxacillin was observed. However, 27.4\% resistance to clarithromycin was detected, although this is comparable with previous Scottish data [30]. The absence of notable antimicrobial resistance from these data could suggest that current hospital infection control and MRSA screening policies are effective. However, decreases in antimicrobial resistance have also previously been associated with changes in the dominance of the circulating bacterial strain [31].

This study was hindered by an inability to access more relevant clinical data. This represents a wider limitation of clinical research that encounters the use of discrete databases and systems for different clinical utilities. A further limitation is the potential for intrinsic bias because of individual patients contributing multiple results, although these most likely represented different infectious episodes from the same foot ulcer. We considered addressing this by removing duplicate results from individual patients but deemed that the greatest clinical relevance was provided by the data as presented. Provision of multiple results may therefore have also introduced a degree of bias into the antimicrobial resistance data as, for genetic or behavioural reasons, some patients may be more prone to infection with certain bacterial species. Knowledge of the microbiology of diabetic foot infections is important in helping monitor the presence of antimicrobial resistance among this at-risk population and provides evidence to guide the targeting of novel anti-infectives.

\section{Conclusion}

In summary, this retrospective analysis has increased the understanding of the microbiology of DFIs in Scotland, provided reassuring data regarding antimicrobial resistance and suggests that the prevalence of $S$. aureus in DFIs may be associated with increasing patient age.

\section{Abbreviations}

DFIs: Diabetic foot infections; DFUs: Diabetic foot ulcers; DHARMa: Diagnostics for HierArchical Regression Models; MRSA: Methicillin resistant Staphylococcus aureus; NHS: National Health Service; QEUH: Queen Elizabeth University Hospital; UK: United Kingdom

\section{Acknowledgements}

We are grateful for the input of those that peer reviewed this manuscript. JDJ would like to thank his wife for useful discussions and reviewing the manuscript.

\section{Authors' contributions}

Designed the study: JDJ. Collected the data: EC, JEB, GEH, LMLH. Analysed the data: KEM, CYJ. Wrote the manuscript: KEM, JDJ. All authors read and approved the final manuscript.

\section{Funding}

JDJ acknowledges support from the Medical Research Council (MC PC 18046). KEM was supported by a Carnegie Trust Vacation Scholarship. The funders has no role in no role in the design of the study and collection, analysis, or interpretation of data nor in writing the manuscript.

\section{Availability of data and materials}

The datasets generated during and/or analysed during the current study are available from the corresponding author on reasonable request. 


\section{Ethics approval and consent to participate}

This article does not contain any studies with human participants or animals performed by any of the authors. All data was anonymised before use and this study was Caldicott Guardian approved by NHS Greater Glasgow and Clyde.

\section{Consent for publication}

Not applicable.

\section{Competing interests}

The authors declare that they have no competing interests.

\section{Author details}

'Edinburgh Medical School: Biomedical Sciences, Infection Medicine, University of Edinburgh, Chancellor's Building, 49 Little France Crescent, Edinburgh EH16 4SB, UK. 'Biomedical Teaching Organisation, Edinburgh Medical School: Biomedical Sciences, University of Edinburgh, Doorway 3, Teviot Place, Edinburgh EH8 9AG, UK. ${ }^{3}$ Diabetes and Endocrinology, Queen Elizabeth University Hospital, 1345 Govan Road, Govan, Glasgow G51 4TF, UK. ${ }^{4} Z$ ZJU-UoE Institute, Zhejiang University School of Medicine, International Campus, Zhejiang University, 718 East Haizhou Road, Haining, Zhejiang 314400, People's Republic of China.

Received: 29 November 2019 Accepted: 27 February 2020

Published online: 12 March 2020

\section{References}

1. Guariguata L, Whiting DR, Hambleton I, Beagley J, Linnenkamp U, Shaw JE. Global estimates of diabetes prevalence for 2013 and projections for 2035. Diabetes Res Clin Pract. 2014;103(2):137-49.

2. World Health Organization. In: Roglic G, editor. Global report on diabetes. Geneva: World Health Organization; 2016

3. Kwon KT, Armstrong DG. Microbiology and antimicrobial therapy for diabetic foot infections. Infect Chemother. 2018;50(1):11.

4. Mishra SC, Chhatbar KC, Kashikar A, Mehndiratta A. Diabetic foot. BMJ. 2017; 16:j5064.

5. Scottish Diabetes Survey Monitoring Group. Scottish diabetes survey 2017 2017 [cited 2019 Aug 26]. Available from: http://www.diabetesinscotland. org.uk/Publications/SDS\%202017.pdf.

6. Saltoglu N, Yemisen M, Ergonul O, Kadanali A, Karagoz G, Batirel A, et al. Predictors for limb loss among patient with diabetic foot infections: an observational retrospective multicentric study in Turkey. Clin Microbiol Infect. 2015;21(7):659-64

7. Lavery LA, Fontaine JL, Bhavan K, Kim PJ, Williams JR, Hunt NA. Risk factors for methicillin-resistant Staphylococcus aureus in diabetic foot infections. Diabet Foot Ankle. 2014;5(1):23575

8. Eleftheriadou I, Tentolouris N, Argiana V, Jude E, Boulton AJ. Methicillinresistant Staphylococcus aureus in diabetic foot infections. Drugs. 2010; 70(14):1785-97.

9. Stacey HJ, Clements CS, Welburn SC, Jones JD. The prevalence of methicillin-resistant Staphylococcus aureus among diabetic patients: a meta-analysis. Acta Diabetol. 2019;56:907-21.

10. Ray A, Malin D, Nicolau DP, Wiskirchen DE. Antibiotic tissue penetration in diabetic foot infections. J Am Podiatr Med Assoc. 2015;105(6):520-31.

11. Smith K, Collier A, Townsend EM, O'Donnell LE, Bal AM, Butcher J, et al. One step closer to understanding the role of bacteria in diabetic foot ulcers: characterising the microbiome of ulcers. BMC Microbiol. 2016;16(1):54.

12. National Institute for Health and Clinical Excellence. Diabetic foot problems: prevention and management NICE guideline [NG19] [Internet]. [cited 2020 Jan 24]. Available from: https://www.nice.org.uk/guidance/ng19/chapter/ Recommendations\#diabetic-foot-infection.

13. Bates $D$, Mächler M, Bolker B, Walker S. Fitting linear mixed-effects models using Ime4. J Stat Softw. 2015;67(1):1-48.

14. Kuznetsova A, Brockhoff PB, Christensen RHB. ImerTest package: tests in linear mixed effects models. J Stat Softw. 2017;82(1):1-26.

15. Hartig F. DHARMa: residual diagnostics for hierarchical (multi-level/mixed) regression models. [R package version 0.2.4] [Internet]. 2019 [cited 2019 Aug 211. Available from: https://cran.r-project.org/web/packages/DHARMa/ vignettes/DHARMa.html.
16. Chisman R, Lowry D, Saeed MA, Tiwari A, David MD. Prescribing antibiotics in diabetic foot infection: what is the role of initial microscopy and culture of tissue samples? Int Wound J. 2017;14(4):685-90

17. Dang CN, Prasad YDM, Boulton AJM, Jude EB. Methicillin-resistant Staphylococcus aureus in the diabetic foot clinic: a worsening problem. Diabet Med. 2003;20(2):159-61.

18. Tentolouris N, Jude EB, Smirnof I, Knowles EA, Boulton AJM. Methicillinresistant Staphylococcus aureus: an increasing problem in a diabetic foot clinic. Diabet Med. 1999:16(9):767-71.

19. Nelson EA, Wright-Hughes A, Brown S, Lipsky BA, Backhouse M, Bhogal M et al. Concordance in diabetic foot ulceration: a cross-sectional study of agreement between wound swabbing and tissue sampling in infected ulcers. Health Technol Assess. 2016;20(82):1-176.

20. Wertheim HF, Melles DC, Vos MC, van Leeuwen W, van Belkum A, Verbrugh $\mathrm{HA}$, et al. The role of nasal carriage in Staphylococcus aureus infections. Lancet Infect Dis. 2005;5(12):751-62.

21. Gamblin J, Jefferies JM, Harris S, Ahmad N, Marsh P, Faust SN, et al. Nasal self-swabbing for estimating the prevalence of Staphylococcus aureus in the community. J Med Microbiol. 2013;62(3):437-40.

22. Jauneikaite E, Ferguson T, Mosavie M, Fallowfield JL, Davey T, Thorpe N, et al. Staphylococcus aureus colonisation and acquisition of skin and soft tissue infection amongst Royal Marines recruits: a prospective cohort study. Clin Microbiol Infect. 2019 [cited 2019 Aug 26]. https://doi.org/10.1016/j.cmi. 2019.07.014.

23. Becker K, Heilmann C, Peters G. Coagulase-negative staphylococci. Clin Microbiol Rev. 2014;27(4):870-926.

24. Tong SYC, Davis JS, Eichenberger E, Holland TL, Fowler VG. Staphylococcus aureus infections: epidemiology, pathophysiology, clinical manifestations, and management. Clin Microbiol Rev. 2015;28(3):603-61.

25. Hatipoglu M, Mutluoglu M, Uzun G, Karabacak E, Turhan V, Lipsky BA. The microbiologic profile of diabetic foot infections in Turkey: a 20-year systematic review: diabetic foot infections in Turkey. Eur J Clin Microbiol Infect Dis. 2014:33(6):871-8.

26. Lipsky BA, Aragón-Sánchez J, Diggle M, Embil J, Kono S, Lavery L, et al. IWGDF guidance on the diagnosis and management of foot infections in persons with diabetes. Diabetes Metab Res Rev. 2016;32(S1):45-74.

27. Malone M, Bowling FL, Gannass A, Jude EB, Boulton AJM. Deep wound cultures correlate well with bone biopsy culture in diabetic foot osteomyelitis. Diabetes Metab Res Rev. 2013;29(7):546-50.

28. Senneville E, Melliez H, Beltrand E, Legout L, Valette M, Cazaubie M, et al. Culture of percutaneous bone biopsy specimens for diagnosis of diabetic foot osteomyelitis: concordance with ulcer swab cultures. Clin Infect Dis. 2006;42(1):57-62.

29. Mehraj J, Witte W, Akmatov MK, Layer F, Werner G, Krause G. Epidemiology of Staphylococcus aureus nasal carriage patterns in the community. In: Stadler M, Dersch P, editors. How to overcome the antibiotic crisis: facts, challenges, technologies and future perspectives [internet]. Cham: Springer International Publishing; 2016. p. 55-87 [cited 2019 Aug 21]. (current topics in microbiology and immunology). https://doi.org/10.1007/82_2016_497.

30. Lawes T, López-Lozano J-M, Nebot C, Macartney G, Subbarao-Sharma R, Dare CRJ, et al. Turning the tide or riding the waves? Impacts of antibiotic stewardship and infection control on MRSA strain dynamics in a Scottish region over 16 years: non-linear time series analysis. BMJ Open. 2015;5(3): e006596.

31. Wyllie DH, Walker AS, Miller R, Moore C, Williamson SR, Schlackow I, et al. Decline of meticillin-resistant Staphylococcus aureus in Oxfordshire hospitals is strain-specific and preceded infection-control intensification. BMJ Open. 2011;1(1):e000160

\section{Publisher's Note}

Springer Nature remains neutral with regard to jurisdictional claims in published maps and institutional affiliations. 